\title{
Effects of Compressibility on the Performance of a Wave-Energy Conversion Device with an Impulse Turbine Using a Numerical Simulation Technique
}

\author{
A. Thakker and T. S. Dhanasekaran \\ Wave Energy Research Team, Department of Mechanical and Aeronautical Engineering, University \\ of Limerick, Limerick, Ireland
}

M. Takao

Department of Control Engineering, Matsue National College of Technology, Matsue-shi, Shimane, Japan

T. Setoguchi

Department of Mechanical Engineering, Saga University, Saga-shi, Saga, Japan

This article presents work carried out to predict the behavior of a $0.6 \mathrm{~m}$ impulse turbine with fixed guide vanes as compared with that of a 0.6 hub-to-tip ratio turbine under real sea conditions. In order to predict the true performance of the actual oscillating water column (OWC), the numerical technique was fine-tuned by incorporating the compressibility effect. Water surface elevation versus time history was used as the input data for this purpose. The effect of compressibility inside the air chamber and the turbine's performance under unsteady and irregular flow conditions were analyzed numerically. Considering the quasi-steady assumptions, the unidirectional steady-flow experimental data was used to simulate the turbine's characteristics under irregular unsteady flow conditions. The results showed that the performance of this type of turbine is quite stable and that the efficiency of the air chamber and the mean conversion efficiency are reduced by around $8 \%$ and $5 \%$, respectively, as a result of the compressibility inside the air chamber. The mean efficiencies of the OWC device and the impulse turbine were predicted for 1 month, based on the Irish wave climate, and it was found that the total time period of wave

Received 14 May 2003; accepted 14 May 2003.

The authors acknowledge the financial support given by ESBI, Ireland, and also by the Wave Energy Research Team, Department of Mechanical and Aeronautical Engineering, University of Limerick, Limerick, Ireland.

Address correspondence to A.Thakker, University of Limerick, Limerick, Ireland. E-mail: Ajit.Thakker@ul.ie data used is one of the important factors in the simulation technique.

Keywords Impulse turbine, Irregular waves, Numerical simulation,
Wave energy conversion

Over the past two decades, scientists have been investigating and defining various methods of extracting power from wave motion. Some of these devices utilize the principle of an oscillating water column (OWC). The OWC-based wave-energy power plants convert wave energy into low-pressure pneumatic power in the form of bidirectional airflow. Self-rectifying air turbines are used to extract mechanical shaft power, which is further converted into electrical power by a generator (Fig. 1). Two different turbines are currently in use around the world for wave-energy power generation: the Wells turbine, introduced by Dr. A. A. Wells in 1976 (Ragunathan, 1985), and the impulse type of turbine described by some authors (Kim et al., 1988; Setoguchi et al., 2000). Both these turbines are currently in operation in various power plants in Europe, Canada, Australia, and Asia. Currently, the research around the world is focused on improving the performance of both these turbines under a variety of operating conditions.

Predictions concerning the performance of turbines under real sea conditions is important so designers can get a feel for the behavior of the power-converting device under unsteady, irregular flow conditions. This article deals with the performance prediction for a 0.6- $\mathrm{m}$ diameter impulse turbine with fixed guide vanes under such conditions, using numerical simulation. Assuming the quasi-steady conditions, the unidirectional 


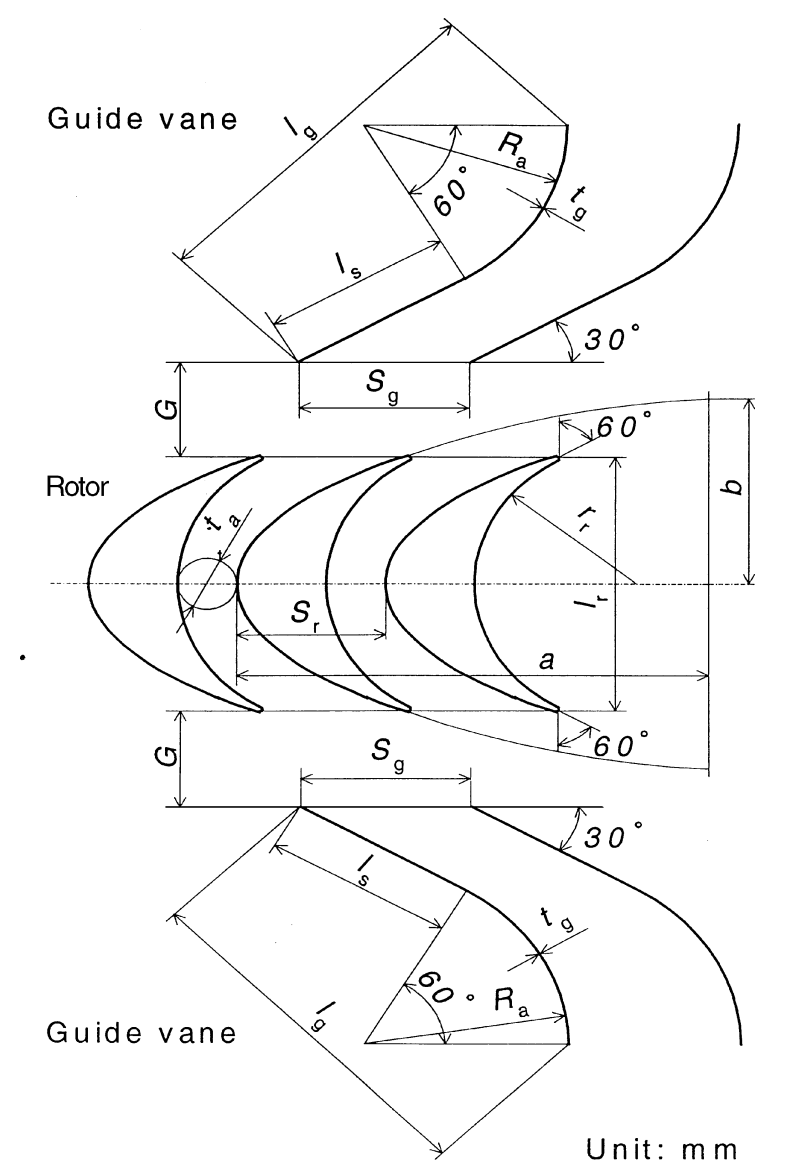

FIGURE 1

Impulse turbine with fixed guide vanes.

steady-flow experimental data were used to simulate turbine performance under varying conditions. Previously, the performance prediction for said turbine had been presented under sinusoidal flow conditions (Thakker et al., 2001). As a next step, the turbine performance was analyzed numerically under simulated irregular and unsteady conditions based on real sea data and assuming that the flow inside the air chamber was incompressible (Setoguchi et al., 2000; Thakker et al., 2001). In the present study, the flow inside the air chamber was considered to be compressible so as to determine the realistic efficiency of such a device and the degree of accuracy of the performance of the turbine under unsteady flow conditions. The simple geometry of the OWC device was used for the simulation. The actual sea data, based on the water surface elevation's time history, was used to simulate the irregular test wave provided by University College Cork, Cork, Ireland (Holmes, 2001).

\section{EXPERIMENTAL ANALYSIS}

A schematic layout of the experimental rig of the Wave Energy Research Team at the University of Limerick is shown in Figure 2. It consists of a bell-mouth entry, a $0.6-\mathrm{m}$ test section with a hub-to-tip ratio of 0.6 , a drive and transmission section,

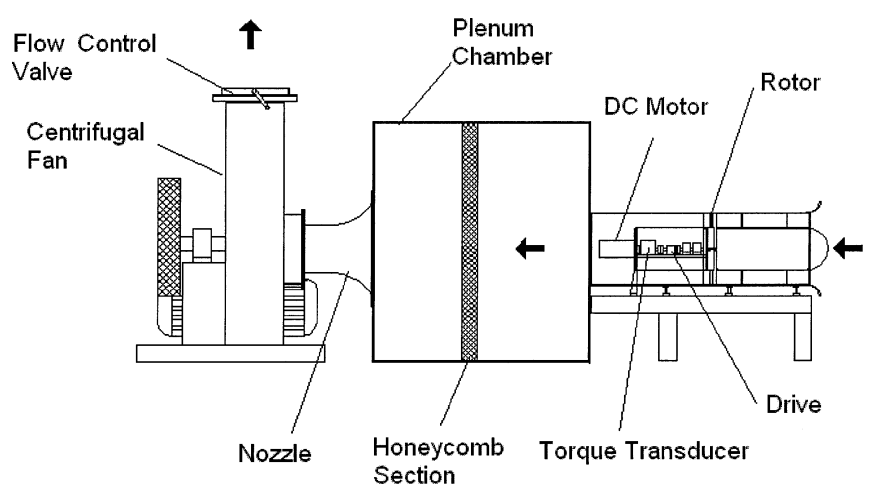

FIGURE 2

Schematic diagram of test rig.

a plenum chamber with a honeycomb section, a calibrated nozzle, and a centrifugal fan. Air is drawn into the open end of the bell-shaped mouth; it passes through the turbine and then enters the plenum chamber. In the chamber, the flow is conditioned and all swirls and vortexes are removed prior to its passing through a calibrated nozzle and finally exiting at the fan outlet. Using a valve at the fan exit controls the flow rate. Details of the test rig's calibration can be found in the work of Thakker and colleagues (2001). The turbine is mounted on a shaft in a cylindrical annular duct with a blade tip clearance of $1 \mathrm{~mm}$. The shaft is coupled to a motor and generator via a torque meter. The two guide vanes were mounted on the upstream and downstream hubs of the rig. The turbine was tested by keeping a constant axial velocity of $8.49 \mathrm{~m} / \mathrm{sec}$. Data were collected by varying the rotational speed from $1250 \mathrm{rpm}$ to $125 \mathrm{rpm}$, thus giving a flow coefficient range of 0.27 to 2.7 under unidirectional steady-flow conditions. The Reynolds number based on the blade chord length was $0.74 \times$ $10^{5}$ at peak efficiency. The specifications of the turbine are listed in the Table 1. The overall performance of the turbines was evaluated experimentally in terms of the angular velocity of the turbine, $\omega$; the torque generated, $T$; the flow rate, $Q_{t}$; and the total pressure drop, $\Delta P$, across the rotor. The results were expressed in the form of the torque coefficient, $C_{T}$; the input power

\section{TABLE 1}

Specification of the turbine

\begin{tabular}{ll}
\hline Blade profile: & \\
Number of blades & $z=30$ \\
Tip diameter & $D=598.0 \mathrm{~mm}$ \\
Chord length & $I_{r}=100.0 \mathrm{~mm}$ \\
Pitch & $S_{r}=50.0 \mathrm{~mm}$ \\
Blade inlet angle & $\gamma=60^{\circ}$ \\
Guide vanes profile: plate type & \\
Pitch & $S_{g}=58.0 \mathrm{~mm}$ \\
Chord length & $l_{g}=131.0 \mathrm{~mm}$ \\
Number of guide vanes & $G=26$ \\
Guide vanes inlet/outlet angle & $\theta=30^{\circ}$ \\
\hline
\end{tabular}




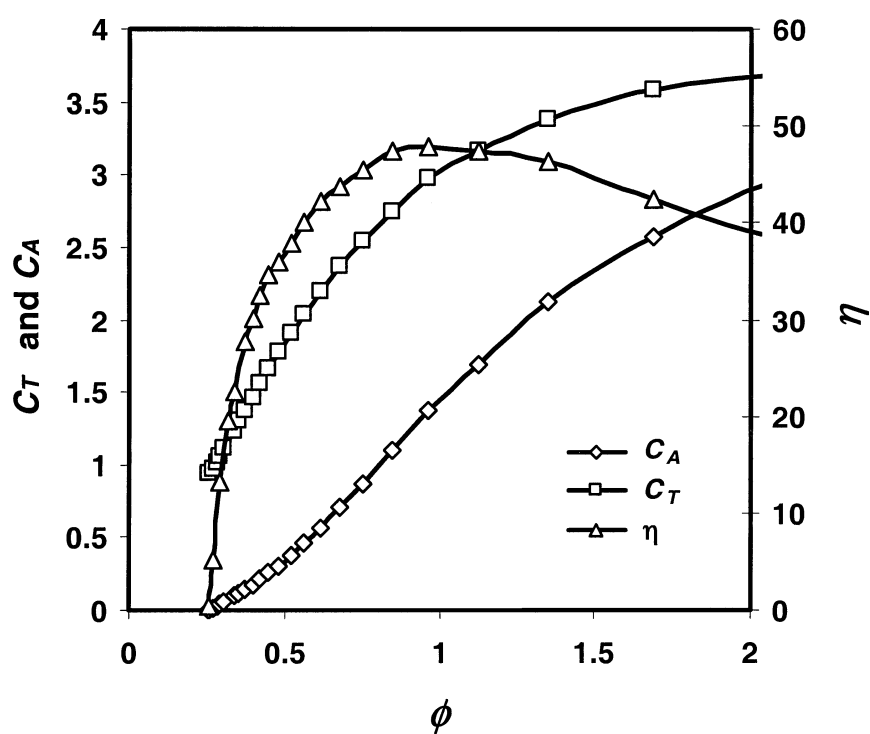

FIGURE 3

Experimental curves of the turbine.

coefficient, $C_{A}$; and the efficiency, $\eta$, in terms of the flow coefficient, $\phi$ (Fig. 3). The definitions used are as follows:

$$
\begin{aligned}
C_{A} & =\Delta p Q_{t} /\left\{\rho_{a}\left(v_{a}^{2}+U_{R}^{2}\right) b l_{r} z v_{a} / 2\right\} \\
C_{T} & =T_{o} /\left\{\rho_{a}\left(v_{a}^{2}+U_{R}^{2}\right) b l_{r} z r_{R} / 2\right\} \\
\eta & =T_{o} \omega / \Delta p Q_{t}=C_{T} /\left(C_{A} \phi\right) \\
\phi & =v_{a} U_{R}
\end{aligned}
$$

\section{PERFORMANCE UNDER IRREGULAR, UNSTEADY FLOW}

The conditions faced by the turbine in an actual wave energy power plant are irregular and unsteady due to the random nature of sea waves. So the performance of the $0.6 \mathrm{~m}, 0.6$ hub-to-tip ratio impulse turbine with fixed guide vanes was evaluated using standard numerical simulation techniques under such conditions. Considering quasi-steady flow conditions, typical turbine characteristics shown in the unidirectional experimental data for said turbine were used for this simulation. The numerical simulation techniques used by Inoue and colleagues (1998) and Setoguchi and colleagues (2000) for predicting the performance of 0.3-m diameter Wells and impulse turbines under irregular flow conditions were adopted for these analyses. This technique was further enhanced by using actual sea data, as will be discussed in later sections. A simple OWC device with a width of $10 \mathrm{~m}$ and a turbine-duct-area-to-air-chamber-area ratio of $m=$ 0.00181 was adopted for the simulation, as shown in Figure 4. The test waves had a mean time period, $T_{s}=6.5 \mathrm{sec}$, and a significant wave height, $H_{s}=2.091 \mathrm{~m}$. For this simulation, 30 waves were used, with a total time span of $164 \mathrm{sec}$. A plot of the nondimensional wave height $H^{*}$ versus nondimensional time $t^{*}$ is shown in Figure 5.

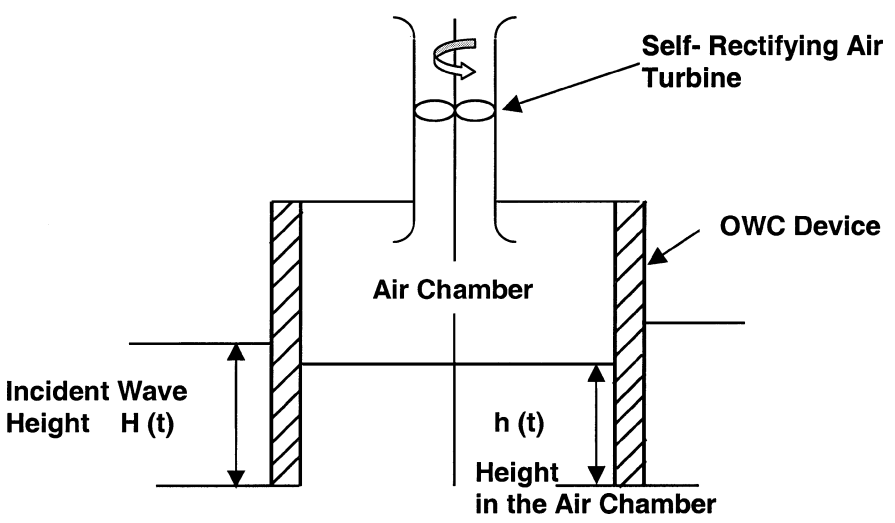

FIGURE 4

Schematic layout of a simple OWC.

\section{Efficiency of the OWC Device}

As mentioned earlier, the main emphasis of this work was on the performance analysis of the impulse turbine under irregular, unsteady flow conditions, with the effects of compressibility taken into account in the OWC. Hence, Equation (5), the air mass flow rate through the turbine given by Sarmento and colleagues (1987), has been reformed as Equation (8) to determine the actual pressure drop in the air chamber:

$$
\dot{m}=-\frac{\mathrm{d}}{\mathrm{d} t}\left[\rho_{c} V\right] .
$$

From the isoentropic relation, $P \rho_{c}^{-\gamma}=P_{a} \rho_{a}^{-\gamma}$,

$$
\rho_{c}=\left(\rho_{a} / \gamma P_{a}\right)(1+p)
$$

where $\rho_{c}$ is linearized with respect to $p$ :

$$
V=V_{o}-A_{c} h
$$

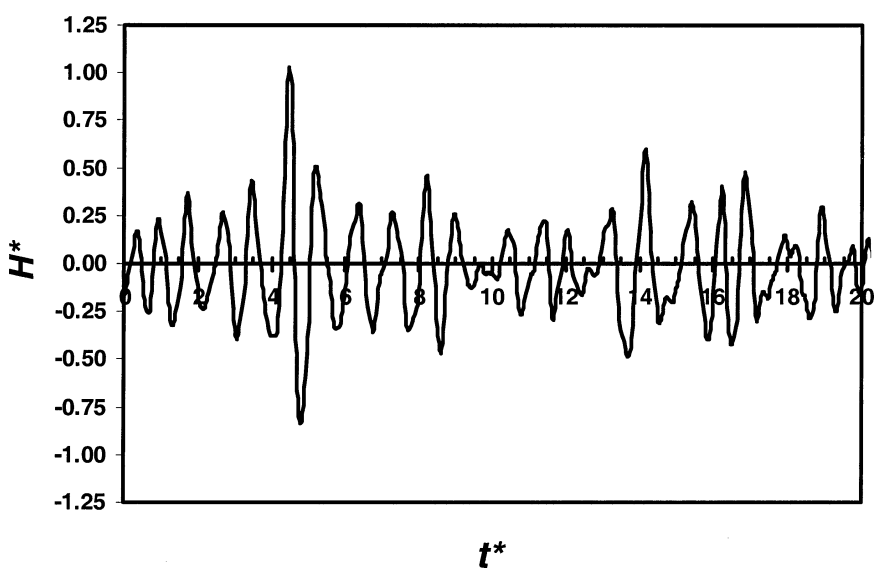

FIGURE 5

Nondimensional wave height $H^{*}$ versus nondimensional time $t^{*}$. 
By substituting Equations (6) and (7) in Equation (5), and assuming $p$ and $h$ are small, we get the following eqnarray:

$$
Q_{t}=Q-\frac{V_{o}}{\gamma p} \frac{\mathrm{d} p}{\mathrm{~d} t}
$$

where $Q=A_{c}(d h / d t)$ and $Q_{t}=A_{t} v_{a}$.

In the above equation the two unknowns are $h$ and $p$. Hence, another equation has been used; it shows the relationship between the incident wave height $(H)$ and the wave height in the air chamber $(h)$, given by Setoguchi and colleagues (1999) as

$$
\frac{\mathrm{d}}{\mathrm{d} t}\left(\rho_{s} h A_{c} \frac{\mathrm{d} h}{\mathrm{~d} t}\right)=\left\{\rho_{s} g(H-h)-\Delta p\right\} A_{c} .
$$

Because $v_{a}=Q_{t} / A_{t}, \Delta p$ is a function of $Q_{t}$ if the rotational speed $U_{R}$ is given. Initially, the relationship between $\Delta p$ and $\mathrm{d} h / \mathrm{d} t$ can be obtained from the $C_{A}-\phi$ characteristics of the turbine (Setoguchi et al., 2000); therefore, we assumed that $\Delta p / \rho_{s}=F\left(Q_{t}\right)$, and Equation (9) can be rewritten as

$$
h \frac{\mathrm{d}^{2} h}{\mathrm{~d} t^{2}}+\left(\frac{\mathrm{d} h}{\mathrm{~d} t}\right)^{2}+F\left(Q_{t}\right)-g(H-h)=0 .
$$

The above equation has been used for the initial iterations to get the approximate value of $h$, and it has applied the value in Equation (9) to determine the value of $\Delta p$. Equations (8) and (9) have been solved simultaneously with the value of $\Delta p$ by applying standard numerical simulation techniques. A RungeKutta-Fehlberg algorithm was employed using MatLab to solve the above equations. The mean incident wave power, $P_{i}$, and the mean pneumatic power in the OWC device, $P_{o}$, are defined as $P_{i}=0.5 H_{s}^{2} T_{s}$ where

$$
P_{I}=W P_{i}
$$

and

$$
P_{O}=\Delta p Q_{t} .
$$

Then the conversion efficiency of the OWC device can be defined as the ratio between the incident wave power and the pneumatic power inside the OWC device:

$$
\bar{\eta}_{c}=P_{O} / P_{I}
$$

\section{Efficiency of the Turbine}

The nondimensional axial velocity through the turbine duct, $v_{a}^{*}$, has been calculated from Equation (8). The running characteristics of the turbine under irregular, unsteady flow conditions can be simulated by using the steady-flow characteristics of a turbine at a constant rotational speed by assuming quasi-steady flow conditions. The use of quasi-steady conditions has been validated by previous studies on the Wells turbine (Inoue et al., 1998) and the impulse turbine (Setoguchi et al., 1993). The mean output $C_{o}$ and the mean input coefficient $C_{i}$ can be defined, respectively, as

$$
\bar{C}_{o}=\frac{1}{t^{*}} \int_{0}^{t^{*}} C_{T}(\phi)\left(\frac{\left(K \bar{\omega}^{*}\right)^{2}+v_{a}^{*^{2}}}{2}\right) \sigma\left(\frac{4(1-v)}{1+v}\right) \bar{\omega}^{*} \mathrm{~d} t^{*}
$$

and

$$
\bar{C}_{i}=\frac{1}{t^{*}} \int_{0}^{t^{*}} C_{A}(\phi)\left(\frac{\left(K \bar{\omega}^{*}\right)^{2}+v_{a}^{*^{2}}}{2}\right) \sigma\left(\frac{4(1-v)}{1+v}\right) v_{a}^{*} \mathrm{~d} t^{*}
$$

It can be noted from the previous two equations that the performance of the turbine can be calculated as a function of $K \omega^{*}$ and $v_{a}^{*}$ when torque coefficient $C_{T}(\phi)$, input coefficient $C_{A}(\phi)$ solidity $\sigma$, hub-to-tip ratio, and nondimensional angular speed are specified; $\phi=v_{a}^{*} /\left(K \bar{\omega}^{*}\right) ; v_{a}^{*}=m T_{s} v_{a} / H_{s} ;$ and $\bar{\omega}^{*}=\omega T_{s}$. The mean efficiency of the turbine can be defined as

$$
\bar{\eta}_{t}=\bar{C}_{o} / \bar{C}_{i}
$$

Therefore, the mean conversion efficiency for the device at a given constant rotational speed and under irregular, steady-flow conditions can be calculated as

$$
\bar{\eta}=\bar{\eta}_{c} \bar{\eta}_{t}
$$

\section{Methodology}

In the previous section, it was mentioned that the turbine's performance under irregular flow conditions depends on a unique parameter, $k \bar{\omega}^{*}$. This parameter includes characteristic parameters of the irregular waves $\left(H_{s}\right.$ and $\left.T_{s}\right)$, turbine speed $(\omega)$, and dimensions of the turbine and air chamber $\left(r_{R}\right.$ and $\left.m\right)$. Therefore, once an optimum value of $k \bar{\omega}^{*}$ can be determined by numerical simulation for a given test wave and dimensionless turbine characteristics, the combination of optimum design values $\left(m, r_{R}\right.$ and $\omega$ ) can be obtained for a site for which the significant wave height, $H_{s}$, and the mean time period, $T_{s}$, are known (Inoue et al., 1998).

The reciprocal $1 /\left(k \bar{\omega}^{*}\right)$ represents the flow coefficient for ordinary fluid machines. To calculate different $1 /\left(k \bar{\omega}^{*}\right)$ values, unidirectional steady-flow experimental data has been used. The trends in $C_{T}(\phi)$ and $C_{A}(\phi)$ characteristics found in experimental results are used to generate turbine performance data for various constant rotational speeds. The turbine performance data are generated for 11 different constant rotational speeds between $1500 \mathrm{r} / \mathrm{min}$ and $125 \mathrm{r} / \mathrm{min}$ to get a $1 /\left(k \bar{\omega}^{*}\right)$ range between 0.206 and 2.46 .

After generating performance data, the incident wave height is converted into wave height in the chamber for each constant rotational speed, using the Ranga-Kutta-Fehlberg algorithm. Following this conversion, the wave height within the chamber is translated into the volume flow over the turbine, 
using Equation (8) for nondimensional axial velocity. Subsequent calculations lead to the mean turbine output and input characteristics (Equations 14 and 15). Finally, the mean efficiency of the turbine is achieved using Equation (17). Similarly, the efficiency of the OWC device is also calculated for individual $1 /\left(k \bar{\omega}^{*}\right)$ by using Equations (11), (12), and (13). Finally, the overall mean efficiency of the device is determined by using Equation (17) for each constant rotation speed.

\section{RESULTS AND DISCUSSION}

The turbine's performance was evaluated for a wide range of $1 /\left(k \bar{\omega}^{*}\right)$ values, corresponding to a range of constant rotational speeds between $1500 \mathrm{r} / \mathrm{min}$ and $125 \mathrm{r} / \mathrm{min}$. Among them, the maximum overall mean efficiency was found at a rotational speed of $350 \mathrm{r} / \mathrm{min}$ for the given test wave. Therefore, before discussing the overall performance evaluation of the turbine, this case is presented in detail in the following section.

\section{Performance Evaluation at $350 \mathrm{r} / \mathrm{min}$}

The performance of the turbine at a constant rotational speed, $N=350 \mathrm{r} / \mathrm{min}$, considering the flow under unsteady, irregular, and compressible conditions in the air chamber was evaluated numerically. Figures $6 \mathrm{a}$ and $\mathrm{b}$ show the variation in the input coefficient and output coefficient of the turbine, respectively. The present results of the compressibility effect were compared with conditions of incompressible flow (Thakker et al., 2001). From Figure 6a, it is clearly visible that the input flow coefficient to the turbine decreased considerably due to the compressibility effect. This may be because of the pressure rise inside the OWC device, which was suppressed by the compressibility. Further, from the figure it can be seen that the output coefficient was almost unaffected.

The conversion efficiency of the OWC device in cases of incompressible and compressible flow is shown in Figure 7. It is evident from this figure that the efficiency of the OWC device was reduced considerably by the compressibility. It can also be observed that the efficiency fluctuated widely with nondimensional time when compared to conditions of incompressible flow. Figure 8 shows the variation in wave height inside the OWC device with nondimensional time. The figure depicts the actual wave height, $h$, inside the OWC device; the numerical prediction assumed the flow was an incompressible flow, overpredicting throughout the time period considered for this study. Hence, this effect was reflected in terms of pressure drop and considerably reduced efficiency of the OWC device (Fig. 7).

\section{Overall Performance Evaluation with and Without Compressibility}

Figures $9 \mathrm{a}, \mathrm{b}$, and $\mathrm{c}$ show the conversion efficiency of the OWC device, the efficiency of the turbine, and the mean conversion efficiency, respectively, in the cases of compressible flow. In all these figures, the results in cases of incompressibility are
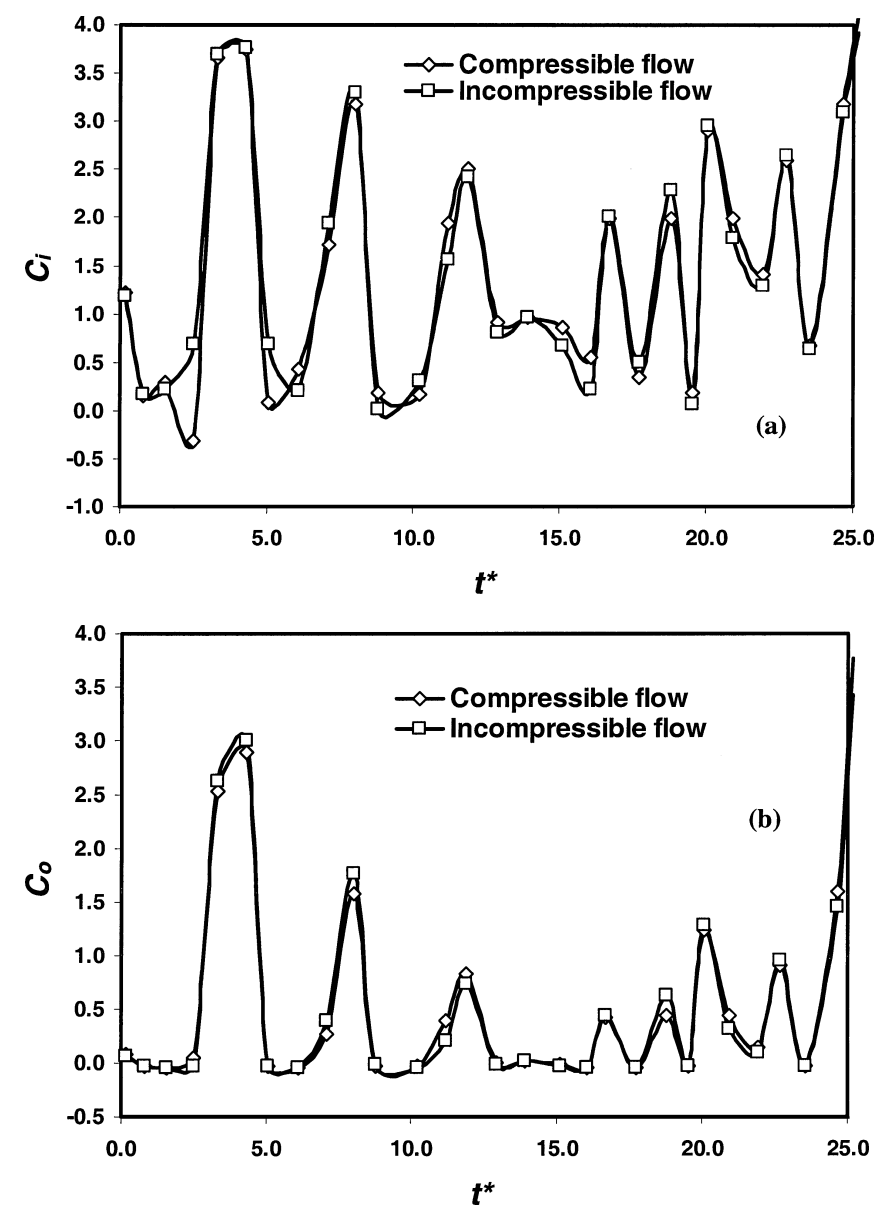

FIGURE 6

Variation of (a) input and (b) output coefficient at $350 \mathrm{r} / \mathrm{min}$.

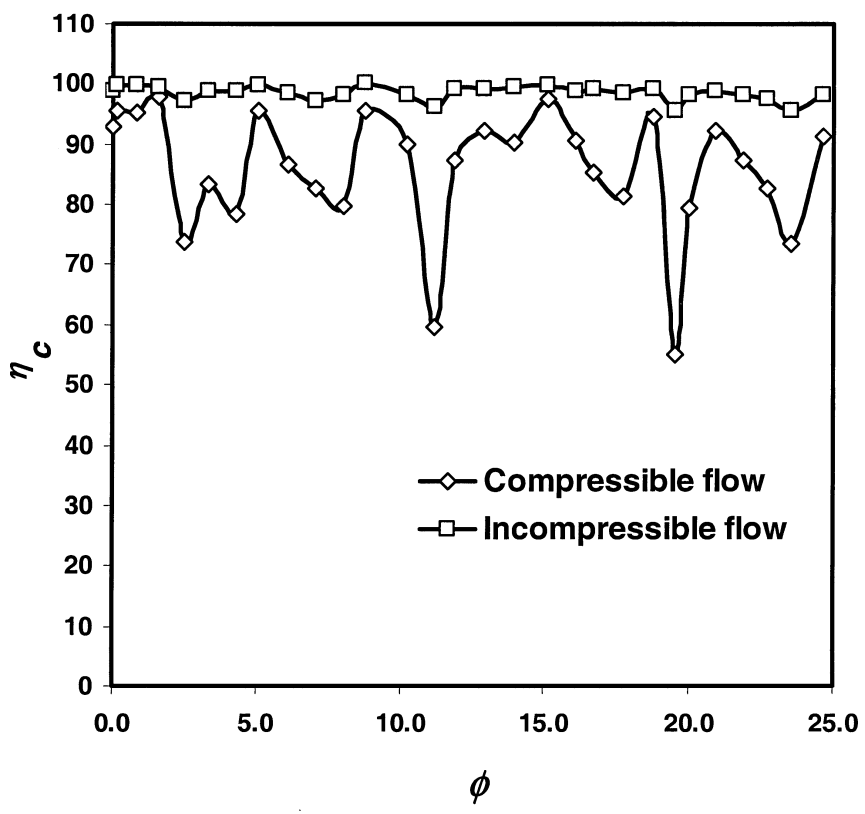

FIGURE 7

Conversion efficiency of OWC device at $350 \mathrm{r} / \mathrm{min}$. 


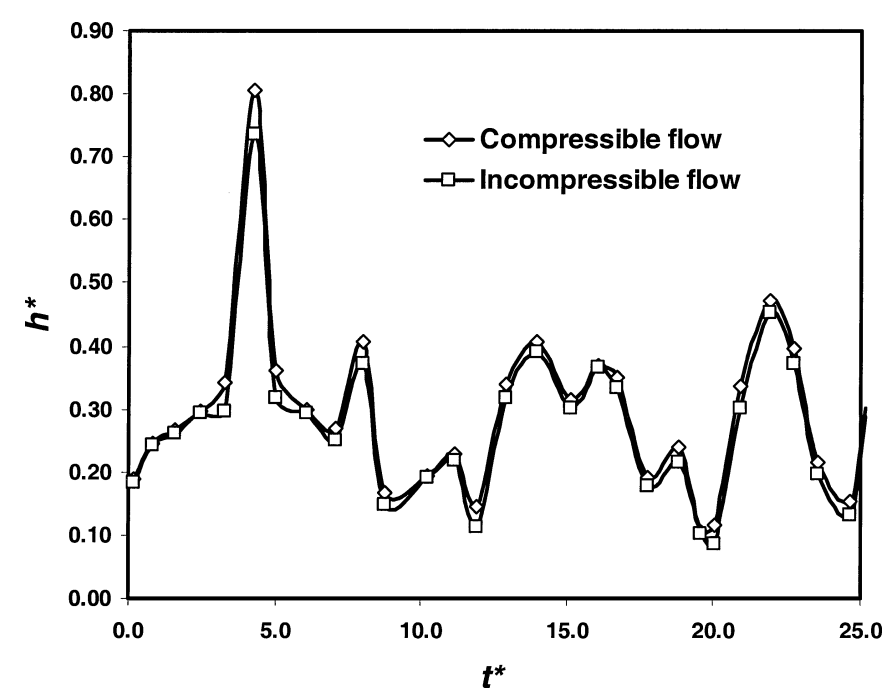

FIGURE 8

Variation of nondimensional height inside the OWC device at $350 \mathrm{r} / \mathrm{min}$.

included for the purpose of comparison. In Figure 9a, it is shown that there is drastic reduction in the efficiency of the OWC device, when the flow inside the air chamber was considered to be compressible. This is approximately 8 percentage points less than in the case of incompressible flow. Figure $9 \mathrm{~b}$ shows the efficiency of the turbine under unsteady and irregular flows, along with unidirectional steady flow. It can be observed that even though there is considerable decrease in efficiency due to the compressibility effect in the OWC device, the turbine shows stable characteristics during irregular flow for conditions of both incompressible and compressible flow. It can also be noted that the efficiency curve in the compressible case is slightly shifted toward the right side because of reduced axial velocity at the inlet to the turbine for the same wave height, $H$. However, this trend occurs up to certain flow coefficients only; after certain flow coefficients, the turbine experiences nearly same efficiency in both cases. The mean conversion efficiency in both cases is shown in Figure 9c. It can be noted from the figure that 5 percentage points of mean conversion efficiency were lost due to compressibility at peak efficiency. The reduction in efficiency is negligible in low coefficients but increases up to a flow coefficient of 1.5 and remains constant beyond this value.

\section{Overall Performance of the Turbine and OWC Device with Various Wave Data}

The performance of the impulse turbine and the OWC device was predicted by the current numerical technique, which included the compressibility effect on various wave data during various total time periods. Figure 10a shows the efficiency of the OWC device in waves 1 and 2, where waves 1 and 2 represent the wave data for a total time period of $164 \mathrm{sec}$ and 1 month, respectively. In the figure it can be seen that the mean efficiency of the OWC device is around $96 \%$ in wave 2 , whereas in the
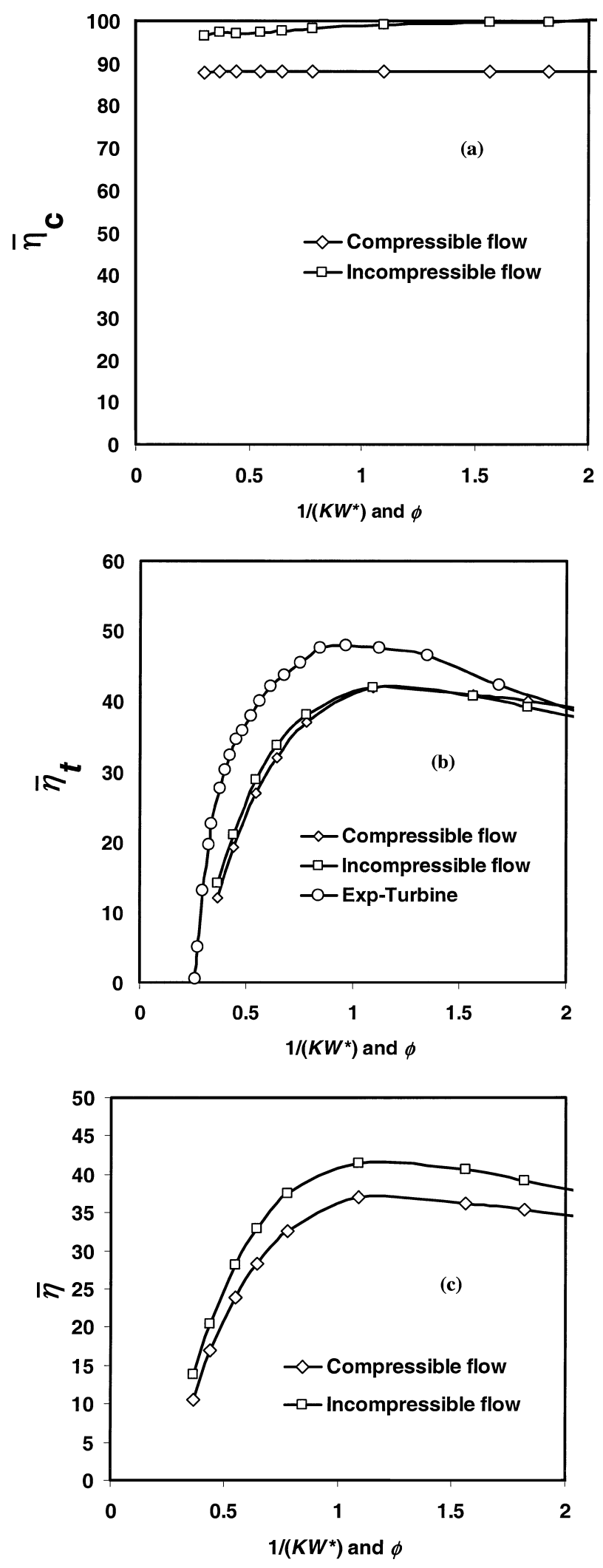

FIGURE 9

Efficiencies with and without compressibility: (a) OWC device; (b) turbine; (c) mean conversion. 

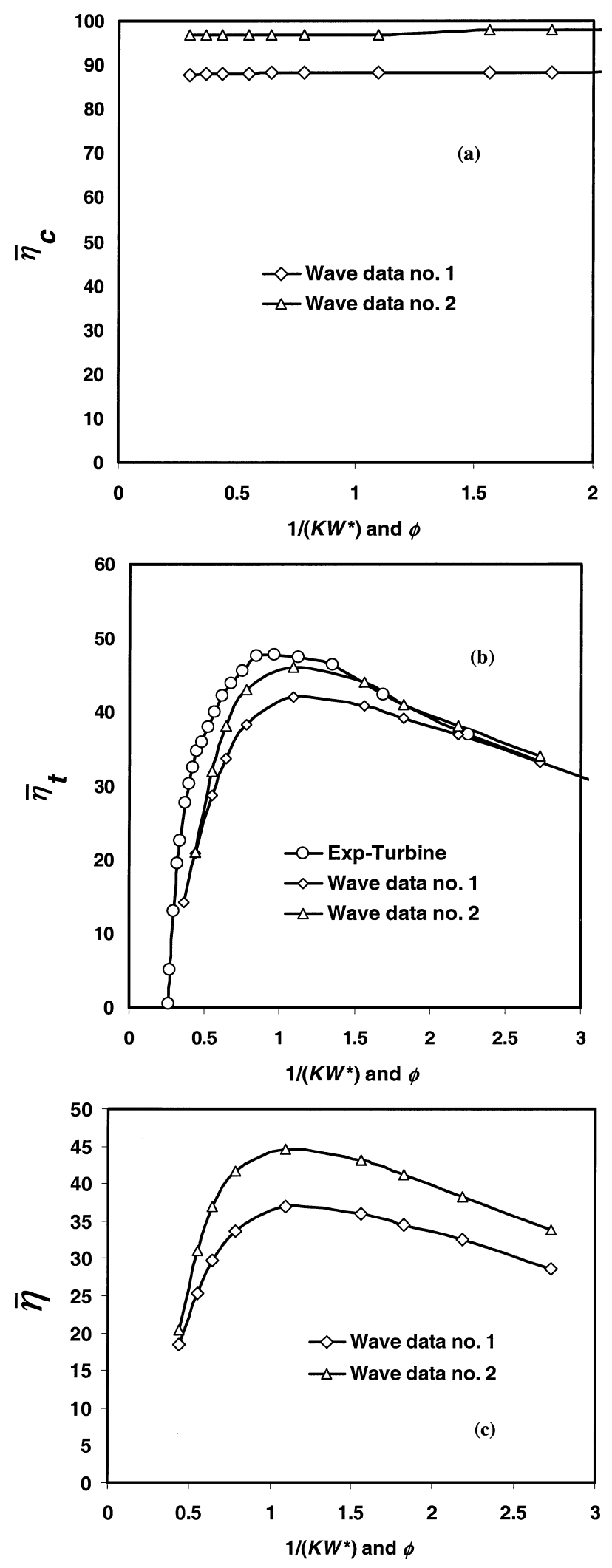

FIGURE 10

Efficiencies with two sets of wave data: (a) OWC device; (b) turbine; (c) mean conversion. case of the prediction for wave 1 , it is around $87 \%$. This may be due to the higher significant wave height at the end of the month in the case of wave 2; hence, the mean efficiency improved in comparison to the case of wave 1 . The efficiency of the turbine in the presence of various wave data is shown in Figure $10 \mathrm{~b}$; the substantial difference in the efficiency of the turbine in combination with various wave data shows the importance of the total time period of the sample wave data. Figure 10c shows the mean conversion efficiency predicted for waves 1 and 2 comparatively. It can be clearly seen in the figure that the mean conversion efficiency under irregular conditions is comparable to that of the impulse turbine's efficiency under the steady-flow condition.

\section{CONCLUSIONS}

The effect of compressibility inside the OWC device and the turbine's performance under unsteady and irregular flow conditions has been analyzed numerically; it reflects the true behavior of an actual OWC device. The results show that there is considerable reduction in the efficiency of an OWC device performance due to compressibility. On the other hand, the turbine performance remains almost unchanged in cases of incompressible and compressible flows. Also, it is found that the turbine shows stable characteristics under irregular, unsteady flow conditions; it is evident that the turbine is capable of converting raw sea waves into useful energy. As the present numerical technique includes the effect of compressibility, it shows that the technique can be used as an indicative tool to predict turbine performance under irregular and unsteady flow conditions. The mean conversion efficiency is reduced by around 5\% by compressible flow inside the OWC device. Furthermore, the performances of the impulse turbine and the OWC device have been predicted for an irregular flow condition for 1 month, based on the Irish wave climate, and it shows that the overall conversion efficiency is comparable to the efficiency of the turbine, which operates under the steady-flow condition.

\section{NOMENCLATURE}

$C_{A} \quad$ Input coefficient

$C_{T} \quad$ Torque coefficient

$\Delta p \quad$ Pressure drop

$G \quad$ Gap between rotor and guide vane

$H_{s} \quad$ Significant wave height

$\mathrm{H} \quad$ Incident wave height

$h \quad$ Water depth in OWC device

$l_{g} \quad$ Chord length of guide vane

$l_{r} \quad$ Chord length of rotor blade

$m$ Area ratio

$p \quad$ Air fluctuation inside chamber

$P \quad$ Pressure inside chamber

$P_{a} \quad$ Atmospheric pressure

$Q \quad$ Volume flow displaced by free surface

$Q_{t} \quad$ Volume flow rate through turbine 
Re Reynolds number

$r_{R} \quad$ Mid-span radius

$\mathrm{r} / \mathrm{min} \quad$ Revolution per minute

$S_{r} \quad$ Rotor blade pitch

$S_{g} \quad$ Guide vane pitch

$t \quad$ Time

$T \quad$ Torque

$T_{S} \quad$ Mean period of incident wave

$U_{R} \quad$ Circumferential velocity at $r_{R}$

$V \quad$ Volume of air inside chamber

$v_{a} \quad$ Axial flow velocity

$W \quad$ Width of chamber

$z \quad$ Number of rotor blades

$\phi \quad$ Flow coefficient

$\gamma \quad$ Specific heat ratio

$\eta \quad$ Turbine efficiency under steady flow

$v \quad$ Hub-to-tip ratio

$\theta \quad$ Setting angle of fixed guide vane

$\rho_{a} \quad$ Density of air

$\rho_{c} \quad$ Density of air inside OWC device

$\omega$ Angular velocity of turbine rotor

\section{REFERENCES}

Holmes, B. 2001. Hydraulics and Maritime Research Centre, University College Cork, Ireland. [Personal communication.]

Inoue, M., Kaneko, K., Setoguchi, T., and Saruwatari, T. 1998. Studies on the Wells turbine for wave power generation (turbine characteristics and design parameters for irregular wave). JSME International Journal 31:676-682.

Kim, T. W., Kaneko, K., Setoguchi, T., and Inoue, M. 1988. Aerodynamic Performance of an Impulse Turbine with Self-Pitch-
Controlled Guide Vanes for Wave Power Conversion. Proceedings of the 1st KSME-JSME Thermal and Fluid Engineering Conference 2:133-137.

Raghunathan, S. 1985. Performance of the Wells self-rectifying turbine. The Aeronautical Journal 89:369-379.

Sarmento, A. J. N. A., Gato, L. M. C., and Falcao, A. F. de O. March 1987. Wave-energy absorption by an OWC device with blade-pitchcontrolled air-turbine. Proceedings of the 6th International Symposium on Offshore Mechanics and Artic Engineering 2:465-473. Houston, TX.

Setoguchi, T., Takao, M., Kinoue, Y., Kaneko, K., and Inoue, M. 2000. Comparative study of performance of turbines for wave power conversion. Proceedings of the 10th International Offshore and Polar Engineering Conference 1:351-357. Seattle, Washington.

Setoguchi, T., Takao, M., Kinoue, Y., Kaneko, K., Santhakumar, S., and Inoue, M. 1999. Study of an Impulse Turbine for Wave Energy Conversion. Proceedings of the 9th International Offshore and Polar Engineering Conference 1:180-187. Brest, France.

Setoguchi, T., Takao, M., Kinoue, Y., Kaneko, K., Santhakumar, S., and Inoue, M. 2000. Study on an impulse turbine for wave energy conversion. International Journal of Offshore and Polar Engineering 10:355-362.

Setoguchi, T., Kaneko, K., Maeda, H., Kim, T. W., and Inoue, M. 1993. Impulse turbine with self-pitch-controlled guide vanes for wave power conversion: Performance of mono-vane type. International Journal of Offshore and Polar Engineering 3:73-78.

Thakker, A., Frawley, P., Khaleeq, H. B., Ansari, A. R., Setoguchi, T., and Takao, M. May 2002. Performance prediction of an impulse turbine under sea conditions using numerical simulation techniques. Proceedings of the 12th International Offshore and Polar Engineering Conference 1:674-681. Kitakyushu, Japan.

Thakker, A., Khaleeq, H. B., and Ansari, A. R. 2001. Numerical simulation of $0.6 \mathrm{~m}$ impulse turbine for wave power conversion under different flow conditions. Proceedings of the 11th International Offshore and Polar Engineering Conference 1:634-637. 

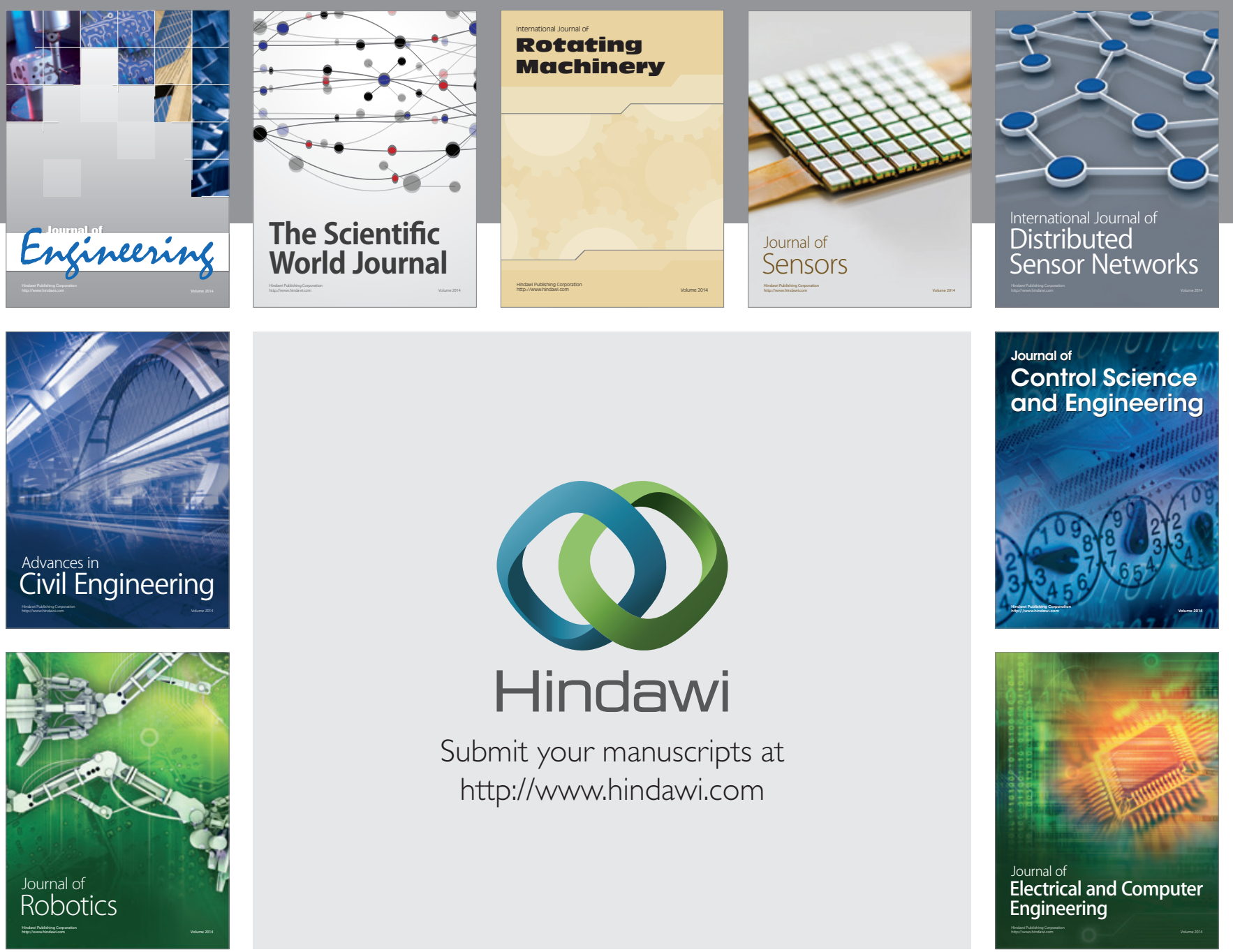

Submit your manuscripts at

http://www.hindawi.com
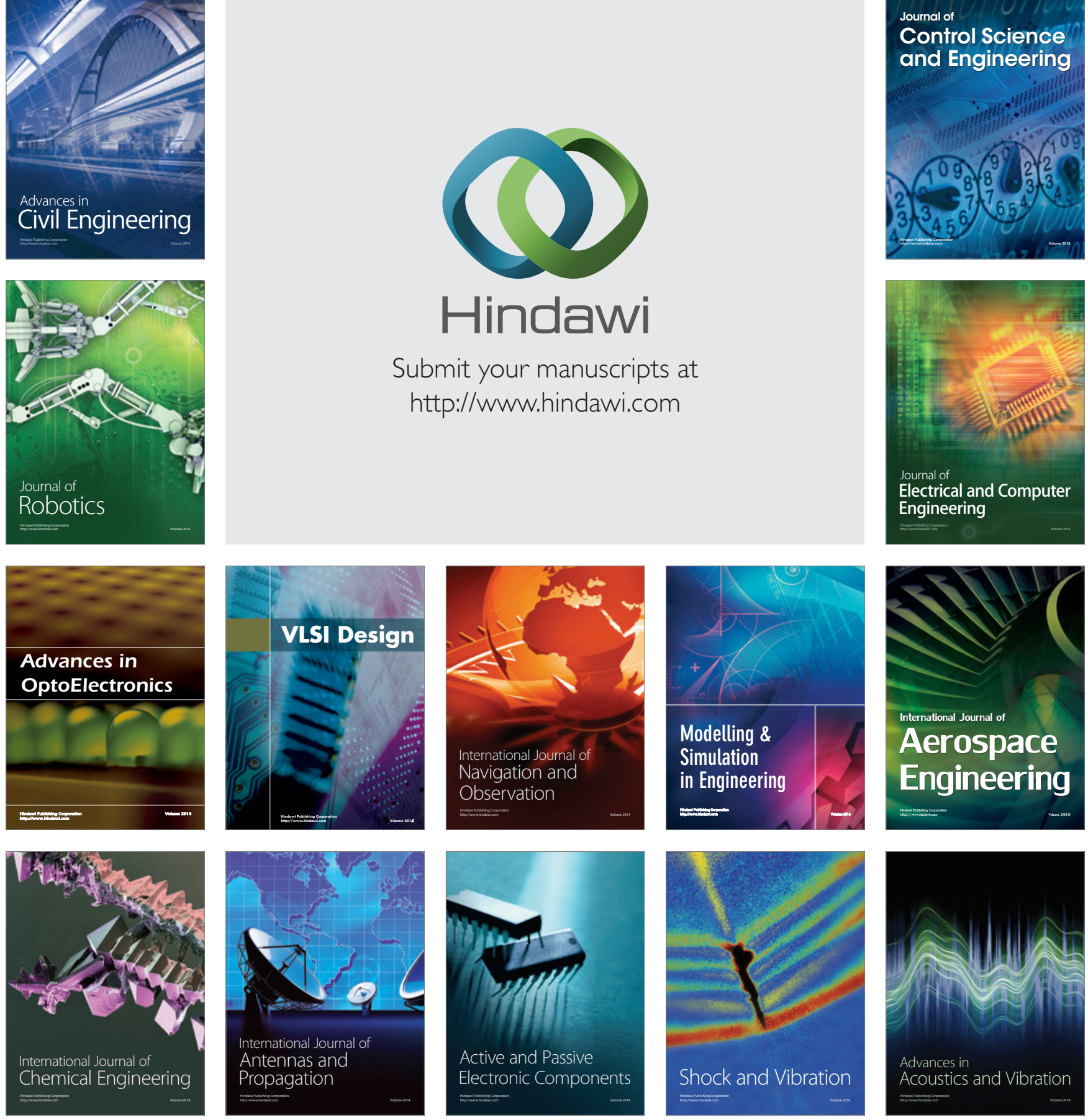